\title{
Author Correction: Imaging emergent heavy Dirac fermions of a topological Kondo insulator
}

Harris Pirie (D), Yu Liu, Anjan Soumyanarayanan (D), Pengcheng Chen, Yang He, M. M. Yee, P. F. S. Rosa, J. D. Thompson, Dae-Jeong Kim, Z. Fisk, Xiangfeng Wang (D), Johnpierre Paglione, Dirk K. Morr (D), M. H. Hamidian (D) and Jennifer E. Hoffman (D)

Correction to: Nature Physics https://doi.org/10.1038/s41567-019-0700-8, published online 11 November 2019.

In the version of this Letter originally published, in Fig. 1a, on the vertical axis, the units 'meV' were incorrect and should have been ' $\mathrm{eV}$ '. The online versions of the Letter have been corrected.

Published online: 4 November 2020

https://doi.org/10.1038/s41567-020-01092-0

๑) The Author(s), under exclusive licence to Springer Nature Limited 2020

\section{Author Correction: High-fidelity entanglement and detection of alkaline-earth Rydberg atoms}

Ivaylo S. Madjarov, Jacob P. Covey, Adam L. Shaw (D), Joonhee Choi, Anant Kale, Alexandre Cooper (D), Hannes Pichler, Vladimir Schkolnik, Jason R. Williams and Manuel Endres (D)

Correction to: Nature Physics https://doi.org/10.1038/s41567-020-0903-Z, published online 25 May 2020.

In Extended Data Table IV originally published for this Article, the values for $P_{11}(2 \pi)$ and $P_{11}^{T}(2 \pi)$ were incorrectly reported as $0.992(2)$ and $0.987(2)$, respectively; they should have been $0.989(2)$ and $0.985(2)$, respectively. The originally reported values were actually those of $1-P_{\{10\}}(2 \pi)$ and $1-P_{\{10\}}^{T}(2 \pi)$, respectively. This error propagated into the calculation of the Bell state fidelities reported in the Article: in the sentence beginning "Finally, using a conservative lower-bound procedure..., ' $\geq 0.983(2)$ ' and ' $\geq 0.995(3)$ ' should have been ' $\geq 0.980(3)$ ' and ' $\geq 0.991(4)$ ', respectively; in the sentence beginning 'With this approach..., '0.983(2)' and '0.995(3)' should have been '0.980(3)' and '0.991(4)', respectively; in the sentence beginning "Furthermore, we estimate a lower bound..., ' $\geq 0.978(2)$ ' and ' $\geq 0.990(3)$ ' should have been ' $\geq 0.975(3)$ ' and ' $\geq 0.987(4)$ ', respectively. Moreover, in Table 1 , in the row 'Bell state fidelity, the uncorrected and SPAM-corrected values of ' $\geq 0.983(2)$ ' and ' $\geq 0.995$ (3)' should have been ' $\geq 0.980$ (3)' and ' $\geq 0.991(4)$ ', and in the row 'Bell state fidelity, T', the uncorrected and SPAM-corrected values ' $\geq 0.978(2)$ ' and ' $\geq 0.990(3)$ ' should have been ' $\geq 0.975(3)$ ' and ' $\geq 0.987(4)$ '. The online versions of the Article have been amended and the Supplementary Information file has been replaced.

Published online: 4 November 2020

https://doi.org/10.1038/s41567-020-01097-9

(c) The Author(s), under exclusive licence to Springer Nature Limited 2020 